Supporting information for

\title{
Poly(lactic acid) Toughening through Chain End Engineering
}

\author{
Kai Li, ${ }^{\text {a }}$ Yu Wang, ${ }^{\text {a }}$ Matthew Rowe,,${ }^{\text {acc }}$ Xianhui Zhao, ${ }^{\text {a }}$ Tianyu Li, ${ }^{\text {d }}$ \\ Halil Tekinalp, ${ }^{\text {b Soydan Ozcan }}{ }^{\mathrm{b}, \mathrm{c}, *}$
}

${ }^{a}$ Chemical Sciences Division, Oak Ridge National Laboratory, 1 Bethel Valley Road, Oak Ridge, Tennessee, 37831, United States

${ }^{b}$ Manufacturing Demonstration Facility, Energy and Environmental Sciences Directorate, Oak Ridge National Laboratory, 2350 Cherahala Blvd, Knoxville, Tennessee 37932, United States ${ }^{c}$ Department of Mechanical, Aerospace, and Biomedical Engineering, University of Tennessee, Estabrook Rd, Knoxville, Tennessee 37916, United States

${ }^{d}$ Department of Materials Science and Engineering, University of Tennessee, Knoxville, Tennessee 37996, United States

* Corresponding author: ozcans@ornl.gov 
(a)

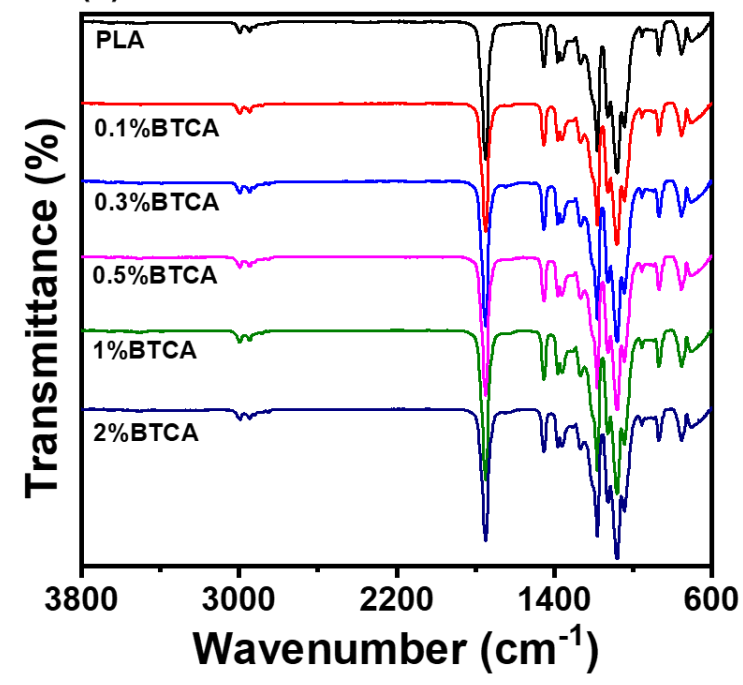

(b)

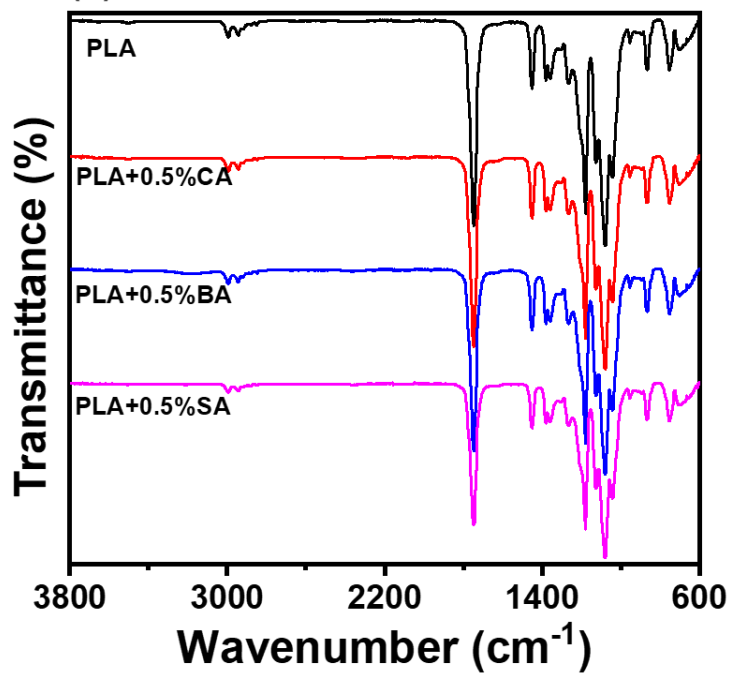

Figure S1. FT-IR spectra of the PLA and BTCA modified samples. (a) PLA+BTCA with different BTCA loadings, (b) CA-, BA-, and SA-modified PLA.

Table S1. Molecular weight of PLA and BTCA modified samples by SEC.

\begin{tabular}{ccc}
\hline Sample & $\mathbf{M n}_{\mathbf{n}}(\mathbf{k D a})$ & Dispersity \\
\hline PLA & 181 & 1.20 \\
PLA+0.1\% BTCA & 289 & 1.21 \\
PLA+0.3\% BTCA & 210 & 1.20 \\
PLA+0.5\% BTCA & 154 & 1.20 \\
PLA+1.0\% BTCA & 132 & 1.19 \\
PLA+2.0\% BTCA & 101 & 1.20 \\
\hline
\end{tabular}




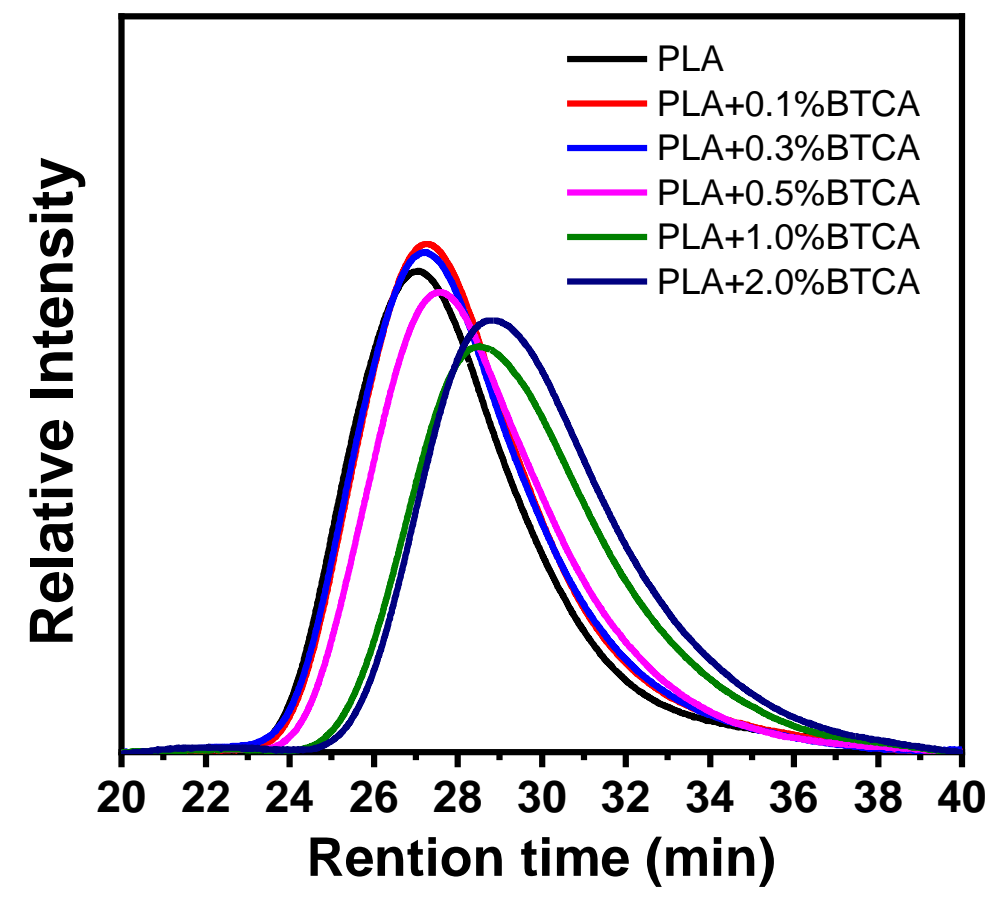

Figure S2. SEC results of PLA and BTCA modified samples obtained from refractive index $(\mathrm{RI})$ detector
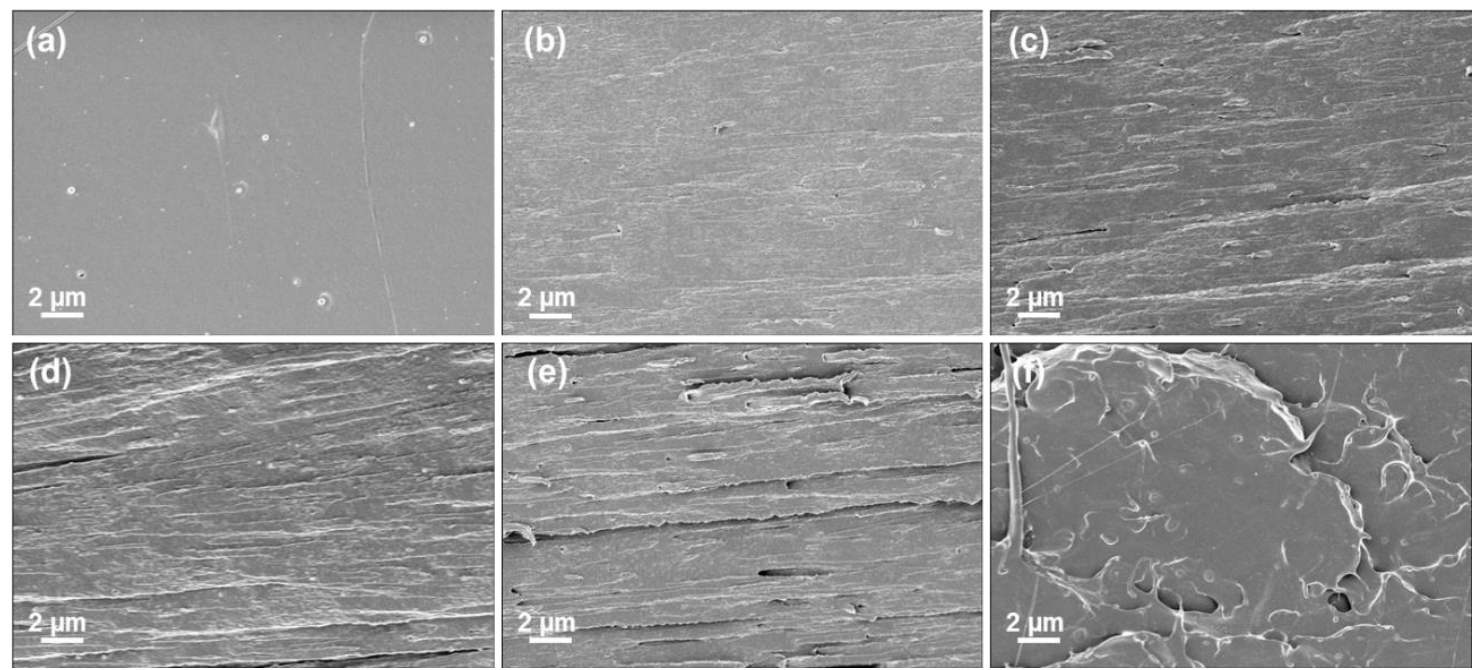

Figure S3. SEM images for PLA and BTCA modified samples after tensile testing. (a) PLA, (b) PLA+0.1\%BTCA, (c) PLA+0.3\%BTCA, (d) PLA+0.5\%BTCA, (e) PLA+1.0\%BTCA, (f) PLA $+2.0 \%$ BTCA. 


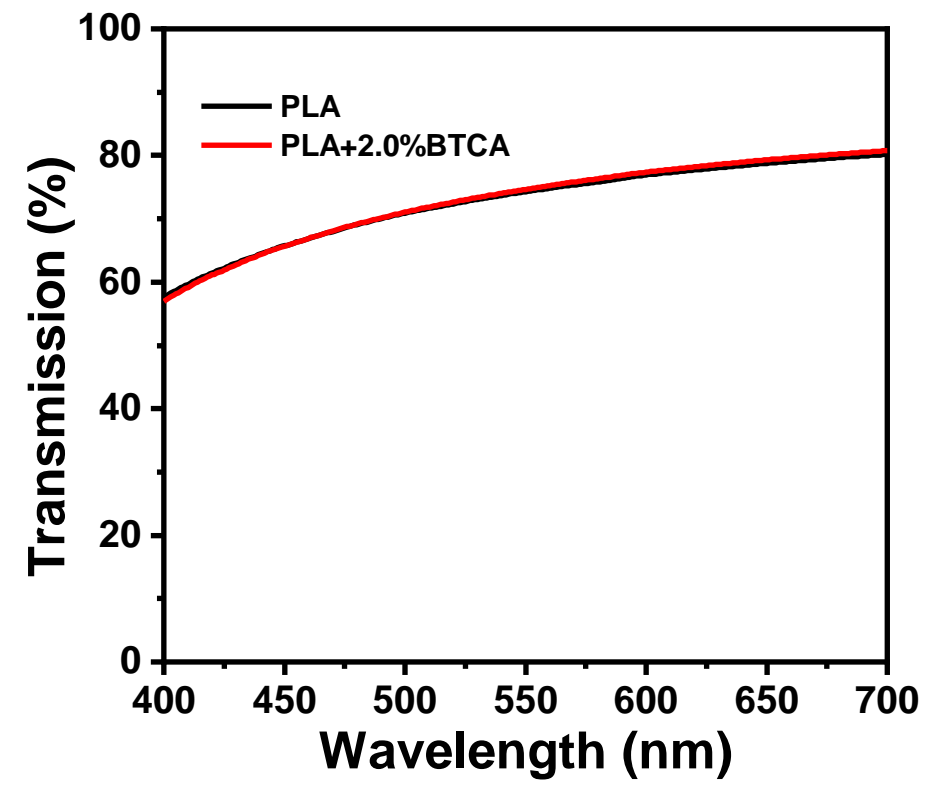

Figure S4. UV-Vis spectra of neat PLA and PLA+2.0\%BTCA.

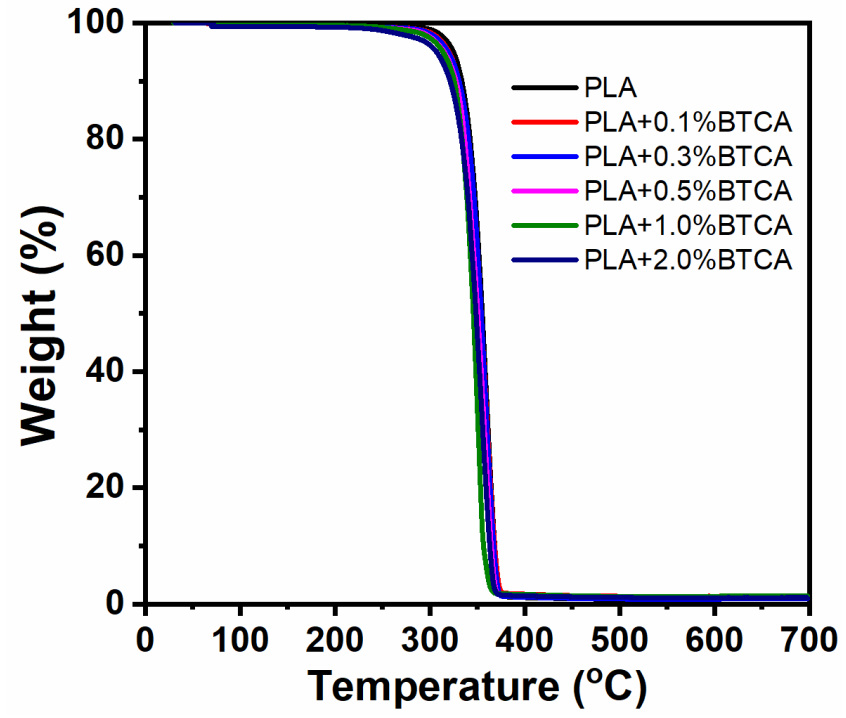

Figure S5. TGA results for PLA+BTCA at various BTCA loadings. 


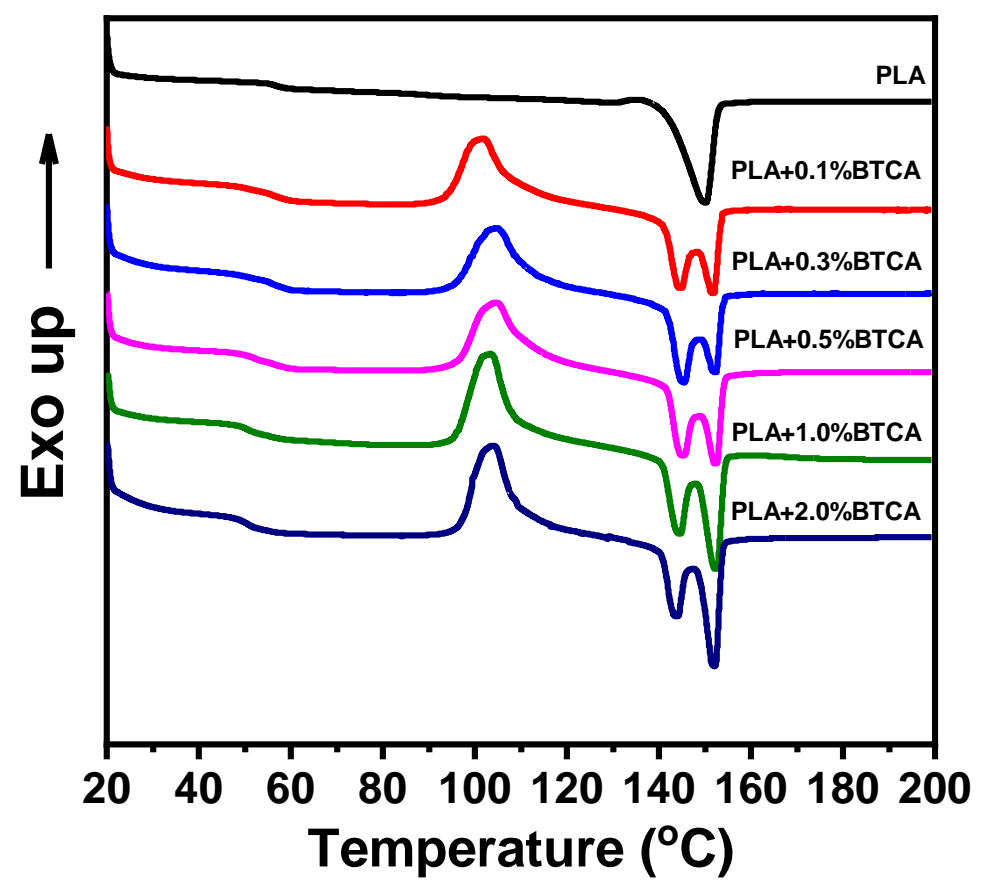

Figure S6. DSC results for PLA composites at various BTCA loadings.

Table S2. Thermal properties of modified PLA. ${ }^{a}$

\begin{tabular}{ccccccc}
\hline Samples & $\mathrm{T}_{\mathrm{g}}\left({ }^{\circ} \mathrm{C}\right)$ & $\mathrm{T}_{\mathrm{c}}\left({ }^{\circ} \mathrm{C}\right)$ & $\mathrm{T}_{\mathrm{m}}\left({ }^{\circ} \mathrm{C}\right)$ & $\Delta \mathrm{H}_{\mathrm{c}}(\mathrm{J} / \mathrm{g})$ & $\Delta \mathrm{H}_{\mathrm{m}}(\mathrm{J} / \mathrm{g})$ & $\chi(\%)$ \\
\hline PLA+0.5\%BA & 51.3 & 93.2 & 150.2 & 11.6 & 22.7 & 11.9 \\
PLA+0.5\%CA & 52.4 & 87.3 & $143.1,152.0$ & 25.0 & 26.7 & 1.9 \\
PLA+0.5\%SA & 52.0 & 94.9 & $143.9,152.6$ & 30.5 & 31.7 & 1.3 \\
\hline
\end{tabular}

Notes: ${ }^{a}$ T5\%: decomposition temperature; $\mathrm{T}_{\mathrm{g}}$ : glass transition temperature; Tc: crystallization temperature; $\mathrm{T}$ m: melting point; $\Delta \mathrm{H}_{\mathrm{m}}$ : melting enthalpies; $\Delta \mathrm{H}_{\mathrm{c}}$ : crystallization enthalpies; $\chi$ : crystallinity. $\mathrm{T}_{5} \%, \mathrm{~T}_{\mathrm{g}}, \mathrm{T}_{\mathrm{c}}, \mathrm{T}_{\mathrm{m}}, \Delta \mathrm{H}_{\mathrm{m}}$, and $\Delta \mathrm{H}_{\mathrm{c}}$ were determined from TGA and DSC. 